\title{
Engineering Anisotropy in Glass with Ultrafast Laser Assisted Nanostructuring
}

\author{
Martynas Beresna, Rokas Drevinskas, Jingyu Zhang, Mindaugas Gecevičius and Peter G. Kazansky \\ Optoelectronics Research Centre, University of Southampton, SO17 1BJ, United Kingdom \\ Author e-mail address: m.beresna@soton.ac.uk
}

\begin{abstract}
Recent applications of femtosecond laser assisted self-assembled nanostructures will be overviewed. Specifically, polarization sensitive optical elements and 5-dimensional optical data storage with practically unlimited life-time will be demonstrated and discussed.

OCIS codes: (140.7090) Ultrafast lasers; (260.6042) Singular optics; (210.0210) Optical data storage
\end{abstract}

Material processing with ultrafast lasers has attracted considerable interest due to new science and a wide range of applications from laser surgery, integrated optics to optical data storage [1,2]. A decade ago it has been discovered that under certain irradiation conditions ordered sub-wavelength structures with features smaller than $20 \mathrm{~nm}$ can be formed in the volume of silica glass [3]. Here we discuss recent applications of self-assembled sub-wavelength structuring specifically polarization sensitive optical elements such as the S-waveplate and polarization multiplexed optical data storage.

Nanogratings exhibits much larger refractive index change than isotropic refractive index increase which is exploited for waveguide writing. For diffractive optics the sign of refractive index does not play crucial role thus a negative refractive index change of nanogratings is well suited for this purpose. Additionally, the femtosecond laser written nanostructure acts as a micro-waveplate with slow axis oriented perpendicular to the polarization of the laser beam [4]. By continuously scanning the bulk of the glass a uniform birefringent layer can be inscribed, which depending on spatial pattern of slow axis can act as a certain optical element, which can control polarization and phase of the passing light (Fig. 1). Using lenses of moderate NA (0.15-0.2) the optical elements with half-wave retardance for $\sim 500 \mathrm{~nm}$ can be written in a single scan at a speed of several millimeters per second. The half-wave retardance is of particular interest since, when circularly polarized light is transmitted through a half-wave plate an absolute phase shift results, which is equal to twice the rotation angle of the wave plate. Theoretically any phase pattern can be achieved solely by means of geometric phase with efficiencies reaching $100 \%$ [5].
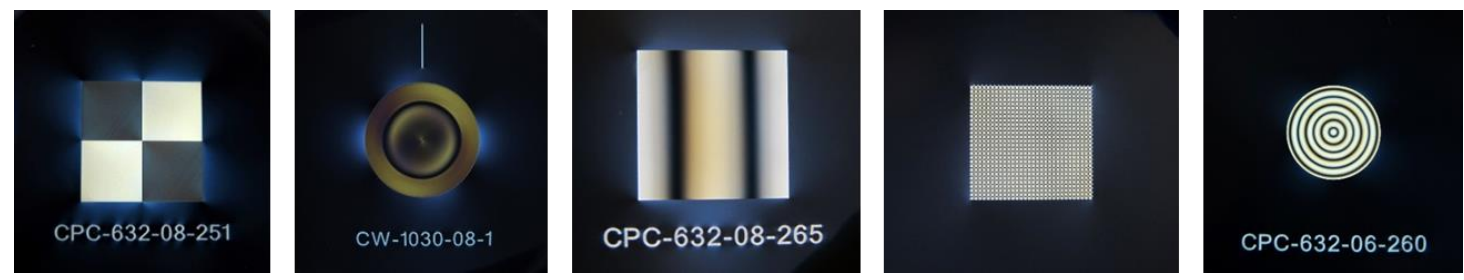

Fig. 1. Polarization sensitive optical elements imaged in the cross-polarized light. Such laser imprinted structures can act as diffraction gratings, lenses, holograms or beam shapers. (Image provided by Workshop of Photonics Ltd.)

The S-waveplates (Southampton-Super-Structured-waveplates) are one of the examples of such birefringent optical elements, which can be used for obtaining axially symmetric polarization state, e.g. radial or azimuthal [6]. Recently, such radial symmetry of the electric field attracted considerable interest due to its ability to produce strong longitudinal component. Alternatively, the same S-waveplate can be used for generating optical beams carrying angular momentum. The ability to control amount of angular momentum by changing polarization of incident beam is one of particular benefits of the S-waveplate. This enabled us to gradually control the torque transferred to microparticles trapped by an optical vortex [7]. We also were able to extend this technique to higher order optical vortices with charges as high as one hundred. The observed asymmetry in rotation speed proposes a technique for measuring the interaction between trapped objects and its environment. Vortices with tunable OAM may allow precise control of particles rotation of any type materials and bio-objects such as DNA molecules measuring not only it is linear but also rotational response of the system. 

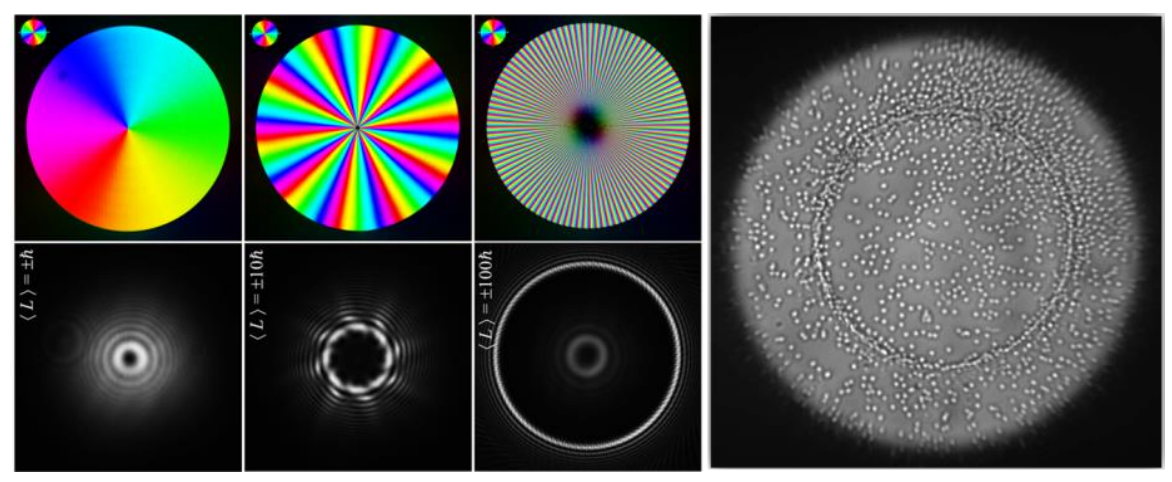

Fig. 2. [Left] S-waveplates for generating optical vortices. The color indicates slow axis orientation of the laser imprinted birefringent layer. The faster is the azimuthal variation of slow axis the higher topological charge can be achieved. Below are given respective intensity distributions of optical vortices with charges 1, 10 and 100. [Right] Silica microparticles suspended in aqueous solution and trapped by $100^{\text {th }}$ charge optical vortex into large rotating ring. Each particle's size is 1 micron.

Another application of femtosecond laser induced nanogratings is optical data storage. The high chemical and thermal resistance of nanogratings makes them an ideal choice for archival data storage with practically unlimited lifetime. The ability to record and read several layers of information via nanogratings was demonstrated by Shimotsuma et al [8]. Later it was demonstrated that digital information can be independently recorded simultaneously in retardance and slow axis orientation [9]. The retardance can be controlled with a precision of about $10 \mathrm{~nm}$, while slow axis angle can be defined with a few degree precision. As a demonstration portraits of two prominent physicists James Clark Maxwell and Isaac Newton were imprinted in the same physical birefringent layer. The portrait of Maxwell was encoded in retardance and Newton's image was recorded in the variation of slow axis orientation. Recently, the digital document was optically encrypted into five dimensions and successfully retrieved by nondestructive quantitative birefringence measurements [10]. The recording time was reduced by two orders of magnitude implementing multiple beams recording technique, where simultaneously up to 100 birefringent dots could be imprinted with variable polarization and intensity (Fig. 1 (right)). Each dot contained 3 bits of information. The information was decoded by combining two binary data sets retrieved from the phase retardance and the slow axis orientation. Out of 11664 bits, which were recorded in three layers, only 42 bit errors were obtained. By recording data with a 1.4 NA objective and shorter wavelength $(250-350 \mathrm{~nm})$, a disc with the capacity of $360 \mathrm{~TB}$ can be recorded. The accelerated aging experiments were performed to evaluate the stability of nanogratings at room temperature and estimate the activation energy of the nanovoids collapse. At room temperature $(303 \mathrm{~K})$ the decay time of nanogratings is $3 \times 10^{20 \pm 1}$ years, indicating unprecedented high stability of nanostructures imprinted in fused quartz.

\section{References}

[1] R.R. Gattass and E. Mazur, "Femtosecond laser micromachining in transparent materials," Nat. Photon. 2, 219-225 (2008).

[2] M. Beresna, M. Gecevičius, and P. G. Kazansky, "Ultrafast laser direct writing and nanostructuring in transparent materials,” Adv. Opt. Photon. 6, 293-339 (2014).

[3] Y. Shimotsuma, P. G. Kazansky, J. Qiu, and K. Hirao, "Self-organized nanogratings in glass irradiated by ultrashort light pulses,” Phys. Rev. Lett. 91, 247405 (2003).

[4] E. Bricchi, B. G. Klappauf, and P. G. Kazansky, "Form birefringence and negative index change created by femtosecond direct writing in transparent materials," Opt. Lett. 29, 119-121 (2004)

[5] N. Yu and F. Capasso, "Flat optics with designer metasurfaces," Nat. Mat. 13, 139-150 (2014).

[6] M. Beresna, M. Gecevičius, and P. G. Kazansky, "Polarization selective elements fabricated by femtosecond laser nanostructuring of glass," Opt. Mat. Express 1, 783-795 (2012).

[7] M. Gecevičius, R. Drevinskas, M. Beresna, and P. G. Kazansky, "Single beam optical vortex tweezers with tunable orbital angular momentum," Appl. Phys. Lett. 104, 231110 (2014).

[8] Y. Shimotsuma et al., "Ultrafast manipulation of self-assembled form birefringence in glass," Adv. Mat. 22, 4039-4043 (2010).

[9] M. Beresna, M. Gecevičius, P. G. Kazansky, T. Taylor, and A. V. Kavokin, "Exciton mediated self-organization in glass driven by ultrashort light pulses," Appl. Phys. Lett. 101, 053120 (2012).

[10] J. Zhang, M. Gecevičius, M. Beresna, and P. G. Kazansky, "Seemingly unlimited lifetime data storage in nanostructured glass," Phys. Rev. Lett. 112, 033901 (2014). 